\section{P188 AN INDEPENDENT EVALUATION OF A NEONATAL END OF LIFE CARE PATHWAY}

Kenda Crozier, ${ }^{1}$ Bruce Lindsay, Jo Wray, ${ }^{2}$ Janet Leeson, ${ }^{3}$ Luaren Andrews ${ }^{3}{ }^{1}$ University of East Anglia, Norwich, UK; ${ }^{2}$ Great Ormond Street Hospital, London: ${ }^{3} E A C H$, Norwich, UK

10.1136/bmjspcare-2011-000105.188

Introduction The neonatal end of life care pathway, implemented in 2008, provides a coordinated planning approach for rapid discharge of babies from Neonatal Intensive Care Unit (NICU) to hospice or home for end of life care.

Aims The evaluation aimed to assess the effectiveness of the neonatal pathway from families' and staff perspectives.

Methods To ensure the evaluation drew on a spread of voices, we recruited participants who had used and provided care pathway. Eight families, 10 internal and 10 external staff were interviewed using a guided conversation technique. Thematic analysis was undertaken and data was further clustered into subthemes.

Findings 42 babies have been referred on the pathway since 2008. Staff and parents expressed a supportive view of the pathway and demonstrated an appreciation for the underlying principles. There is a need for wider understanding of the pathway and care it offers. Bereavement support for families evaluated very well but needs of fathers could be further investigated. The impact on other aspects of hospice activity emerged.

Conclusion The pathway is an innovative service, probably the first in the UK. It represents an increase in service activity of the hospice and contributes to the breadth of service on offer. The experiences of staff and parents outlined in this evaluation should be useful in contributing to the knowledge base about end of life care for very young babies. The knowledge and expertise of staff who have developed and worked with this pathway over the last 3 years should be acknowledged and the model disseminated widely.

Recommendations More regular training opportunities for staff of all levels should focus on case studies rather than simply pathway documentation. Further consideration should be given to managing the demands of respite care when bereaved families are resident. Joint meetings should be used to anticipate and plan. 